\title{
C-Type Natriuretic Peptide/Natriuretic Peptide Receptor 2 Is Involved in Cell Proliferation and Testosterone Production in Mouse Leydig Cells
}

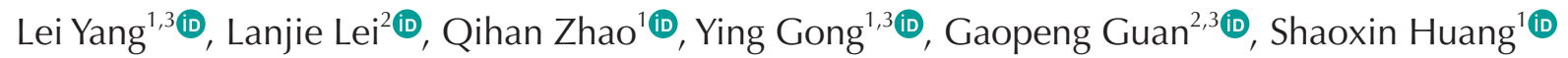 \\ ${ }^{1}$ College of Basic Medical Science, ${ }^{2}$ Clinical Skills Center, Affiliated Hospital of Jiujiang University, ${ }^{3}$ Key Laboratory of System Bio-medicine \\ of Jiangxi Province, Jiujiang University, Jiujiang, China
}

\begin{abstract}
Purpose: This study investigated the role of natriuretic peptide receptor 2 (NPR2) on cell proliferation and testosterone secretion in mouse Leydig cells.

Materials and Methods: Mouse testis of different postnatal stages was isolated to detect the expression C-type natriuretic peptide (CNP) and its receptor NPR2 by quantitative reverse transcription polymerase chain reaction (RT-qPCR). Leydig cells isolated from mouse testis were cultured and treated with shNPR2 lentiviruses or CNP. And then the cyclic guanosine monophosphate production, testosterone secretion, cell proliferation, cell cycle and cell apoptosis in mouse Leydig cells were analyzed by ELISA, RT-qPCR, Cell Counting Kit-8, and flow cytometry. Moreover, the expression of NPR2, cell cycle, apoptosis proliferation and cell cycle related gene were detected by RT-qPCR and Western blot.

Results: Knockdown of NPR2 by RNAi resulted in S phase cell cycle arrest, cell apoptosis, and decreased testosterone secretion in mouse Leydig cells.

Conclusions: Our study provides more evidences to better understand the function of CNP/NPR2 pathway in male reproduction, which may help us to treat male infertility.
\end{abstract}

Keywords: Germ cells; Leydig cells; Testicular diseases; Testosterone

This is an Open Access article distributed under the terms of the Creative Commons Attribution Non-Commercial License (http://creativecommons.org/licenses/by-nc/4.0) which permits unrestricted non-commercial use, distribution, and reproduction in any medium, provided the original work is properly cited.

\section{INTRODUCTION}

Testes, as a critical component of the male reproductive and endocrine tract, the primary functions is to produce sperm and androgens. Mammalian testes consists of several well-defined cell types, including germ cells of different stages, Sertoli cells and Leydig cells.
Each of them is configured with a different location and has specific functions: Germ cells produce sperm and are located in the seminiferous tubules; Leydig cells produce sex hormones and are distributed in the connective tissue of the convoluted seminiferous tubules; Sertoli cells form the basement membrane of the seminiferous tubules and offer the environment neces-

\footnotetext{
Received: May 21, 2018 Revised: Sep 10, 2018 Accepted: Sep 10, 2018 Published online Oct 23, 2018

Correspondence to: Lei Yang iD https://orcid.org/0000-0001-8859-9692

College of Basic Medical Science, Jiujiang University, 17 Lufeng Rd, Xunyang Qu, Jiujiang Shi, Jiangxi Sheng 332000, China.

Tel: +86-0792-8570043, Fax: +86-0792-8577050, E-mail: yangleigeili@163.com

Correspondence to: Shaoxin Huang iD https://orcid.org/0000-0001-9801-4133

College of Basic Medical Science, Jiujiang University, 17 Lufeng Rd, Xunyang Qu, Jiujiang Shi, Jiangxi Sheng 332000, China.

Tel: +86-0792-8570043, Fax: +86-0792-8577050, E-mail: 517159865@qq.com
} 
sary for the differentiation and maturation of germ cells [1]. The importance of Leydig cells in male reproduction is exemplified by the fact that loss of function of gene such as the luteinizing hormone receptor (LHR) [2], insulin-like factor 3 (Insl3) [3], and enzymes involved in testosterone biosynthesis [4].

C-type natriuretic peptide (CNP) and its receptor (natriuretic peptide receptor 2, NPR2) play a role as an oocyte maturation inhibitor in mammalian [5]. Recent studies suggested that CNP can improve oocyte maturation and developmental competence in vitro [6]. Compared with numerous studies about CNP/NPR2 on female reproduction, there is little research about $\mathrm{CNP} /$ NPR2 in male reproduction. Previous studies reported that CNP regulates blood-testis barrier dynamics and is related with spermatogenesis [7]. Sogawa et al [8] investigated phenotype of NPR2-defcient short-limbed-dwarfs mice, and found that the developmental onset and acquisition of spermatogenic function is delayed in NPR2 mutant mice. Subsequently, two studies reported that CNP/NPR2 is related with sperm motility, acrosome reaction and induces sperm attraction for fertilization, thus regulating the reproductive function of males $[9,10]$.

However, the function of CNP/NPR2 in male reproduction remains largely unknown. In this study, we aimed to study the role of CNP/NPR2 on cell proliferation, testosterone secretion and related regulatory mechanisms in mouse Leydig cells.

\section{MATERIALS AND METHODS}

\section{Chemicals and mice}

All chemicals were purchased from Sigma-Aldrich (St. Louis, MO, USA) unless otherwise stated. Male Kunming mice were purchased from the Laboratory Animal Central of Jiujiang University. All mice were fed a typical diet of lab chow and housed in a single room under conditions of constant temperature $\left(25^{\circ} \mathrm{C}-\right.$ $28^{\circ} \mathrm{C}$ ), humidity $(55 \% \pm 5 \%)$ and lighting (12 hours light, 12 hours dark cycle) [11]. All procedures were approved by the Committee for the Ethics on Animal Care and Experiments of Jiujiang University (approval No. SYXK(GAN)2017-0001).

\section{Isolation and cultures of Leydig cells}

Leydig cells were isolated from the testes of 42 - to 49-day-old Kunming mice and cultured as previous report [12]. The purity of Leydig cells was assessed by

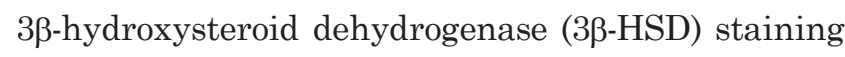
using the modified Wiebe method. Simply, Leydig cells were fixed in $1 \%$ paraformaldehyde for 20 minutes and then washed with phosphate buffer saline (PBS) for three times. After washed the cells were incubated with $1 \mathrm{~mL}$ PBS containing $1 \mathrm{mg}$ bovine serum albumin, $1.5 \mathrm{mg}$ nicotinamide adenine dinucleotide, $0.2 \mathrm{mg}$ nitroblue tetrazolium and $0.25 \mathrm{mg}$ dehydroepiandrosterone for 2 hours at $37^{\circ} \mathrm{C}$ in the dark. Then the cells were gently rinsed with PBS and observed microscopically. During all the culture, the cells were cultured with Dulbecco's modified Eagle's medium/F-12 containing $10 \%$ fetal bovine serum with or without CNP (CNP were used to active CNP/NPR2 signaling pathway), unless otherwise stated.

\section{Transfection of cells with shRNA-} natriuretic peptide receptor 2 lentiviral shRNA-NPR2 and shRNA-negative lentiviral vector were purchased from Genechem (Shanghai, China). The mouse Leydig cells were seeded into 6-well plates, which were cultured to $30 \%$ to $40 \%$ confluence and infected by addition of $1 \times 10^{8} \mathrm{TU} / \mathrm{mL}$ lentivirus, $5 \mu \mathrm{g} / \mathrm{mL}$ polybrene and complete medium. After 12 hours, the lentivirus solution was replaced by complete culture medium and cultured for 36 hours. And the cells were observed under a fluorescence microscope to evaluate the transfection efficiency. The efficiency and specificity of siRNA mediated knockdown were examined by Western blot and quantitative reverse transcription polymerase chain reaction (RT-qPCR). After determine the NPR2 knockdown efficiency, the cells were cultured for subsequent experiments.

\section{Cell proliferation assay}

After culturing with CNP for 24 hours, Cell Counting Kit-8 (CCK8) was added to the cells (10 $\mu \mathrm{L} /$ well). Then the cells were incubated for 1 hour at $37^{\circ} \mathrm{C}$ and measured at $450 \mathrm{~nm}$ by a Microplate Reader (Bio-Rad 680, Hercules, CA, USA). The experiments were performed in triplicate.

\section{Cyclic guanosine monophosphate measurement}

After 30 minutes of treatment, the cells were collected to measure the cyclic guanosine monophosphate (cGMP). The cGMP measurement was according to the procedure described as our previous report [6]. The lev- 
els of cGMP were determined using cGMP-EIA kits obtained from Cayman Chemicals (Ann Arbor, MI, USA). Each sample was measured in triplicate.

\section{Testosterone measurement}

After 24 hours of treatment, mouse Leydig cells were counted. The concentration of testosterone in the culture supernatants $(100,000 \mathrm{cell} / \mathrm{mL}$ culture supernatant) was measured with ELISA kits (Ji Yin Mei; Co. Ltd., Wuhan, China) according to the manufacturer's instructions. Each sample was measured in triplicate.

\section{Cell cycle analysis}

Mouse Leydig cells of the respective experimental groups were collected and fixed in ice-cold $70 \%$ ethanol overnight at $4^{\circ} \mathrm{C}$ after the cells were treatment and count. Then, the cells were washed with PBS and stained with propidium iodide (PI) solution for 20 minutes at $25^{\circ} \mathrm{C}$ in the dark. Finally, the cells were analyzed by flow cytometry using a BD FACS Calibur system and Mod Fit LT for MacV3.0 software. For each analysis, a minimum of 10,000 cells were analyzed. Each sample was measured in triplicate.

\section{Cell apoptosis assay}

The apoptotic rate was determined using the phycoerythrin (PE) and PI double-staining apoptosis analysis kit (Nanjing Key Gen Biotech, Nanjing, China). After washes by PBS for two times, followed by centrifugation, the cells were suspended in $500 \mu \mathrm{L}$ binding buffer. Then, $5 \mu \mathrm{L}$ Annexin PE and PI staining solution were added and incubated at $25^{\circ} \mathrm{C}$ for 10 minutes. The mixture was then analyzed by flow cytometry within 60 minutes. Each sample was measured in triplicate.

\section{RNA isolation and quantitative reverse transcription polymerase chain reaction}

Total RNA was extracted using Trizol reagent (Invitrogen, Carlsbad, CA, USA). First-strand cDNA was synthesized according to the manufacturer's instructions (PrimeScript ${ }^{\circledR}$ RT reagent Kit). RT-qPCR was performed using an ABI StepOnePlus Real-time Detection System (ABI, Foster, CA, USA) and SYBR Green qPCR SuperMix (Invitrogen). Each experiment was repeated independently at least three times, and the fold change in the expression of each gene was analyzed using the $2-^{\Delta \triangle C T}$ method. All the primers were used as Appendix and the $\beta$-actin was used as internal control.

\section{Immunohistochemistry}

Testes of 42- to 49-day-old Kunming mice were fixed in $4 \%$ paraformaldehyde in PBS for one week, dehydrated through a graded ethanol series, and embedded in paraffin. Sections $7 \mathrm{~mm}$ thick were mounted onto glass slides precoated with Poly-L-Lysine Solution and incubated overnight at $37^{\circ} \mathrm{C}$. After dehydrating, samples were placed in citrate buffer $(\mathrm{pH}=6.0)$. Antigen retrieval was performed by treating samples in a microwave oven at $92^{\circ} \mathrm{C}$ for 15 minutes; slides were cooled and then washed in PBS. The sections were pretreated with $3 \%$ (vol $/ \mathrm{vol}$ ) $\mathrm{H}_{2} \mathrm{O}_{2}$ in methanol to quench endogenous peroxidase activity. After being washed with PBS, sections were incubated with $10 \%$ goat serum for 30 minutes at $37^{\circ} \mathrm{C}$. After blocking, sections were incubated overnight at $4^{\circ} \mathrm{C}$ with rabbit polyclonal antibody against NPR2 (Sigma-Aldrich; 1:50 dilutions) in a humidified chamber. After washing, followed by incubation with biotinylated anti-rabbit immunoglobulin $G$ antibody (Beijing 4A Biotech Co., Ltd, Beijing, China) at $37^{\circ} \mathrm{C}$ for 1 hour, and then sections were incubated with horseradish peroxidase-labeled streptavidin at $37^{\circ} \mathrm{C}$ for 30 minutes. Thereafter, positive reactions were visualized with a diaminobenzidine-peroxidase substrate and 30 seconds counterstaining with hematoxylin. Finally, the sections were counterstained with dehydrated and mounted. Slides were imaged using a digital microscope (BA400; Motic, Wetzlar, Germany).

\section{Western blot analysis}

The extraction of cell proteins was used radioimmunoprecipitation assay buffer. Protein determination was performed by the bicinchoninic acid (BCA) assay. $200 \mu \mathrm{g}$ total protein per sample were separated by $12 \%$ sodium dodecyl sulfate polyacrylamide gel electrophoresis and electro transferred to a to polyvinylidene difluoride membrane. After incubation in blocking buffer for 1 hour at room temperature, the membrane was incubated overnight at $4^{\circ} \mathrm{C}$ with the primary antibodies (p-AKT, AKT, $\beta$-actin, 1:1,000, Cell Signaling Technology, Beverly, MA, USA; NPR2, 1:500, SigmaAldrich; Cyp11a1, Star, Bax, Bcl-2, 1:500, Santa Cruz Biotechnology, Santa Cruz, CA, USA). After washing, the membranes were incubated with a secondary antibody conjugated to horseradish peroxidase at $25^{\circ} \mathrm{C}$ for 1 hour. Finally, immunoreactive bands were visualized using a Super Signal West Pico kit according to the manufacturer's (Pierce Biotechnology, Rockford, 


\section{Men's Health}

IL, USA) instructions. The $\beta$-actin was used as internal control and the protein band densities were semiquantified by densitometric analysis using ImageJ (ver. 1.49).

\section{Caspase-3 activity measurement}

Caspase-3 activity was measured using a caspase-3 Activity Colorimetric Assay Kit. Simply, after treatment, the cells were harvested by centrifugation and incubated in lysis buffer on ice for 15 minutes. The lysate was then centrifuged at $15,000 \mathrm{rpm}$ and $4^{\circ} \mathrm{C}$ for 15 minutes, and the protein content was determined using the BCA Protein Assay Kit according to the manufacturer's (Nanjing Biobox Biotech, Naniing, China) instructions. Then, $100 \mathrm{mg}$ of protein in each sample was incubated with the caspase- 3 substrate $(200 \mathrm{mM}$ final concentration) at $37^{\circ} \mathrm{C}$ in a microplate for 4 hours. The samples were measured at $405 \mathrm{~nm}$ using a microplate reader (Bio-Rad 680).

\section{Statistical analyses}

All experiments were replicated at least three times for each group, and the data are presented as the mean \pm standard error of mean. The data were analyzed by ANOVA, followed by Fisher's least significant different test and independent samples Student t-test, with the SPSS software ver. 13.0 (SPSS Inc., Chicago, IL, USA). Differences were considered significant at $\mathrm{p}<0.05$.

\section{RESULTS}

\section{The expression pattern of $\mathrm{C}$-type natriuretic peptide/natriuretic peptide receptor 2 at different postnatal stages of testes}

Previous studies reported that CNP is mainly located and expressed in rat Leydig cells [13,14]. But the expression and location of NPR2 in rodent are still unknown. Here, we first detected the location and expression of NPR2 in adult mouse testes by semiquantitative RTPCR, Western blot and immunohistochemistry. Results showed that NPR2 is mainly expressed in Leydig cells
A

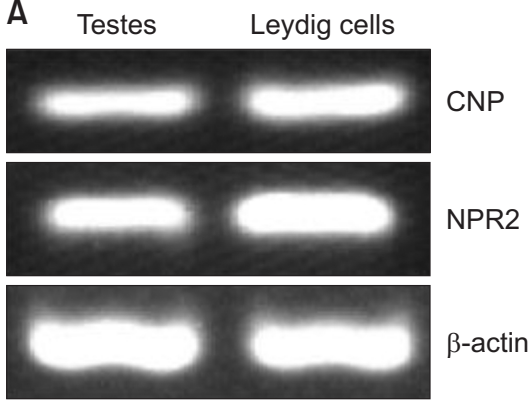

D

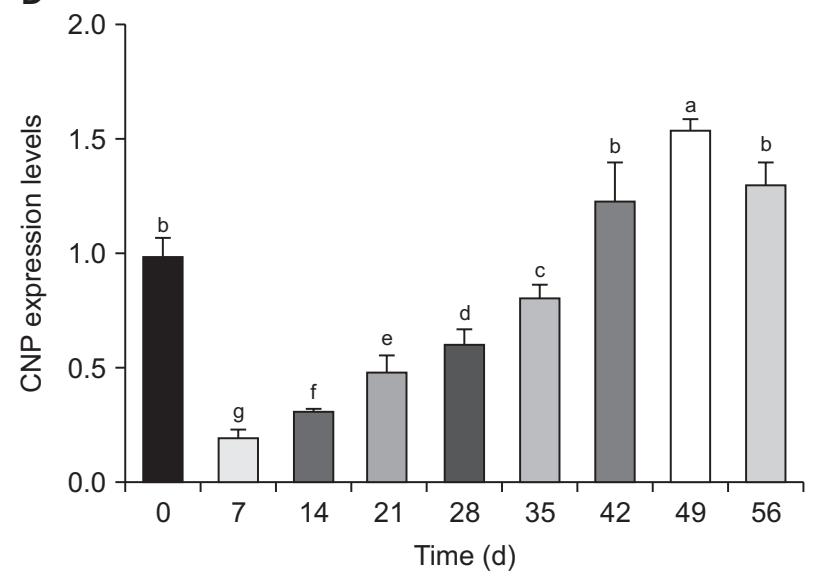

B

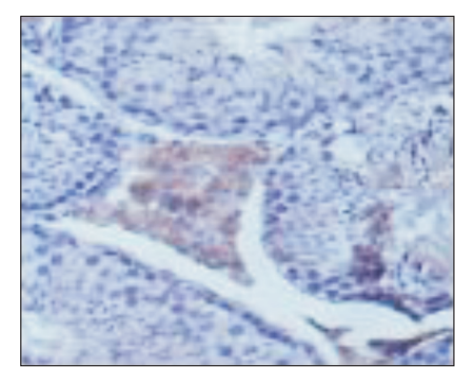

C

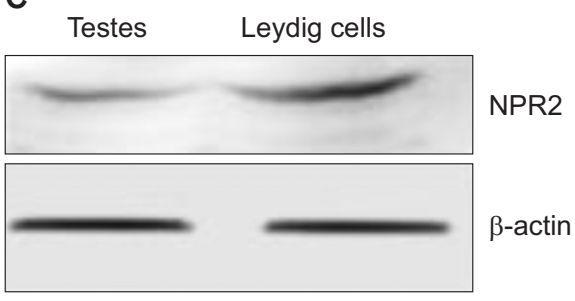

E

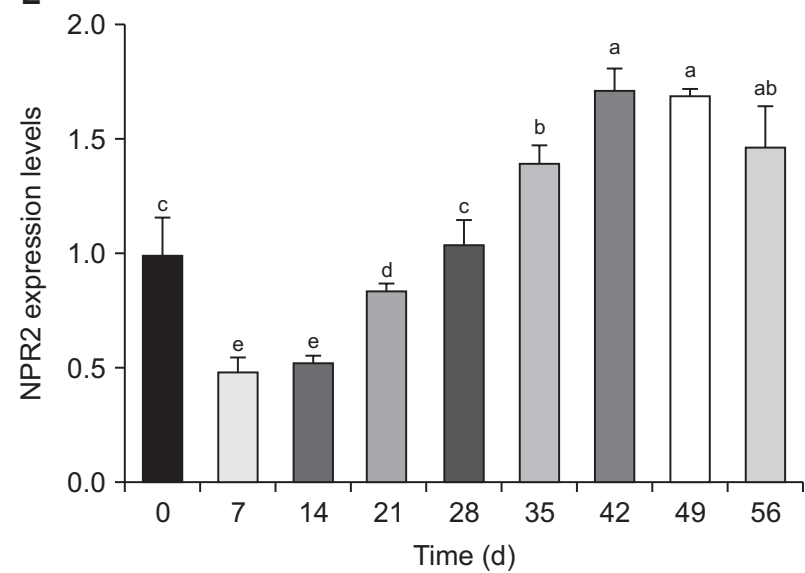

Fig. 1. The expression of C-type natriuretic peptide (CNP)/natriuretic peptide receptor 2 (NPR2) at different postnatal stages of testes. (A) The expression of CNP/NPR2 mRNA in testes and Leydig cells. (B, C) The location by immunohistochemistry $(\times 200)$ and expression by Western blot of NPR2 in testes and Leydig cells. (D) The expression of CNP at mouse different postnatal stages of testes. (E) The expression of NPR2 at mouse different postnatal stages of testes. The $\beta$-actin was used as internal control. The data are presented as the mean \pm standard error of mean of three independent experiments. Bars with different letters are significantly different $(p<0.05)$. 
(Fig. 1A-1C). Moreover, CNP is also mainly expressed in Leydig cells (Fig. 1A). In addition, we detected the expression pattern of CNP and NPR2 at different time of testes after born $(0,7,14,21,28,35,42,49$, and 56 days) by RT-qPCR. Result showed that CNP and NPR2 have a similar expression patterns in the mouse testis at different postnatal stages (Fig. 1D, 1E). The mRNA expression of CNP and NPR2 has a higher expression levels on 0 day, reach its lowest level on postnatal 7 days, and then increases again from 14 to 56 days (Fig. 1D, 1E).

\section{Effects of C-type natriuretic peptide on cyclic guanosine monophosphate production, natriuretic peptide receptor 2 expression, cell proliferation, testosterone secretion in mouse Leydig cells}

To measure the effects of CNP on cGMP production,

A

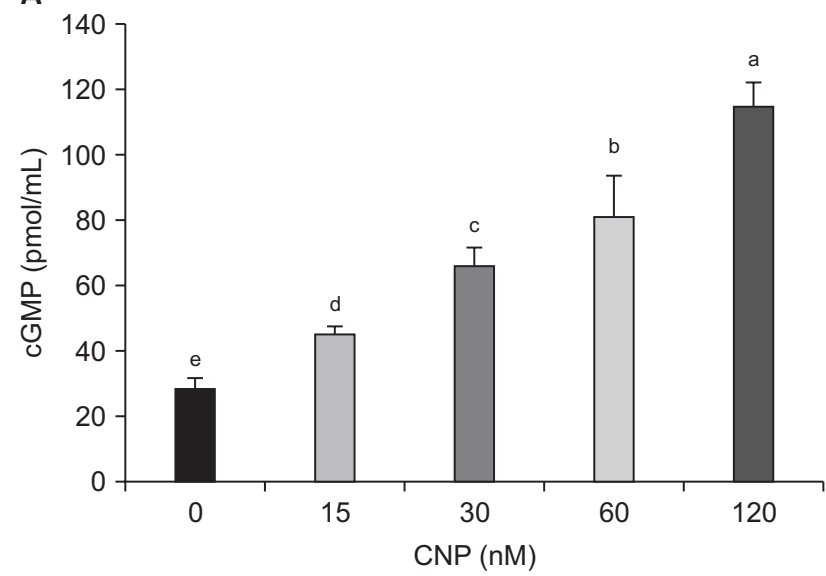

C

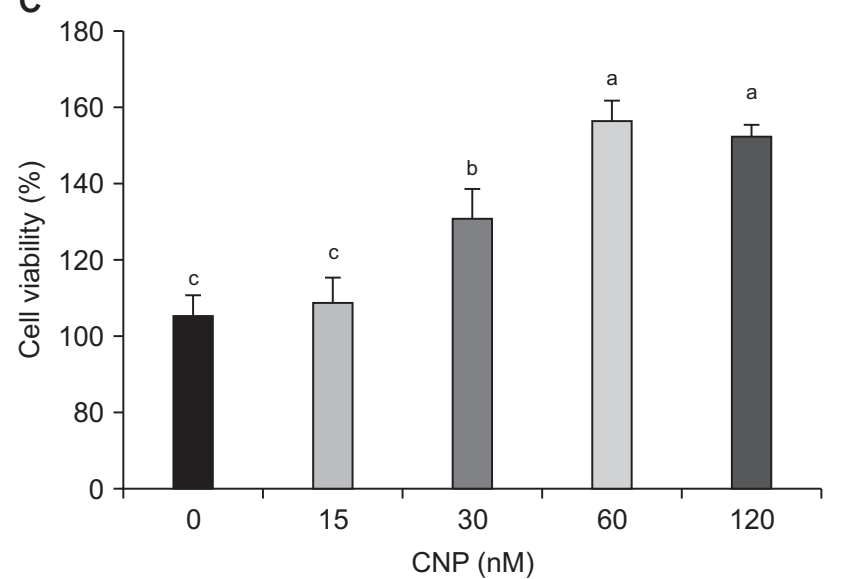

NPR2 expression, cell proliferation, testosterone secretion in mouse Leydig cells, the cells were treated with different concentrations of $\operatorname{CNP}(0,15,30,60,120 \mathrm{nM})$ to measure cGMP production, detect NPR2 expression, cell proliferation and testosterone secretion. The results showed that CNP enhances cGMP production in a dose-dependent manner, and the lowest effective concentration is $15 \mathrm{nM}$ (Fig. 2A). Moreover, RT-qPCR results showed that CNP also increases NPR2 expression in dose-dependent manner and the lowest effective concentration is $30 \mathrm{nM}$ (Fig. 2B). Meanwhile, CCK8 results showed that $\mathrm{CNP}$ enhances cell proliferation in a dose-dependent manner, and the lowest effective concentration is $30 \mathrm{nM}$ (Fig. 2C). In addition, ELISA results showed that CNP promotes the testosterone secretion and the lowest effective concentration is $30 \mathrm{nM}$ (Fig. 2D).
B

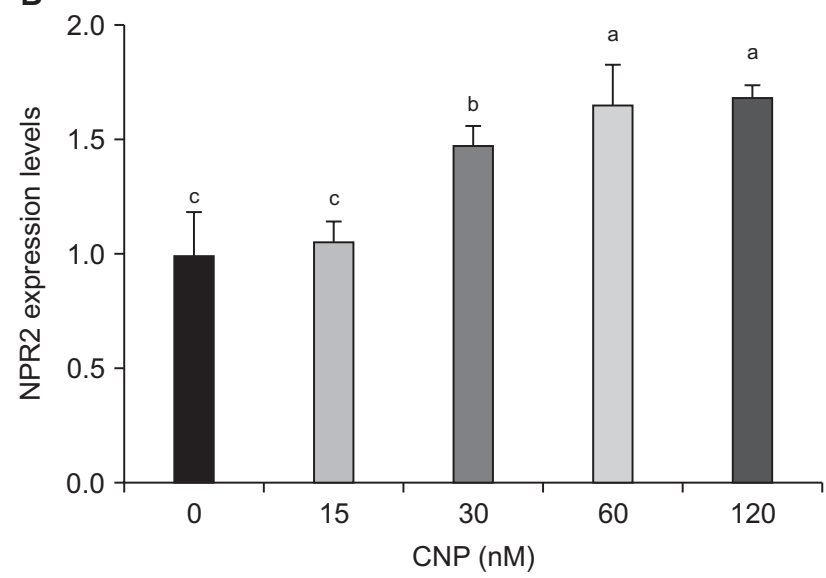

D

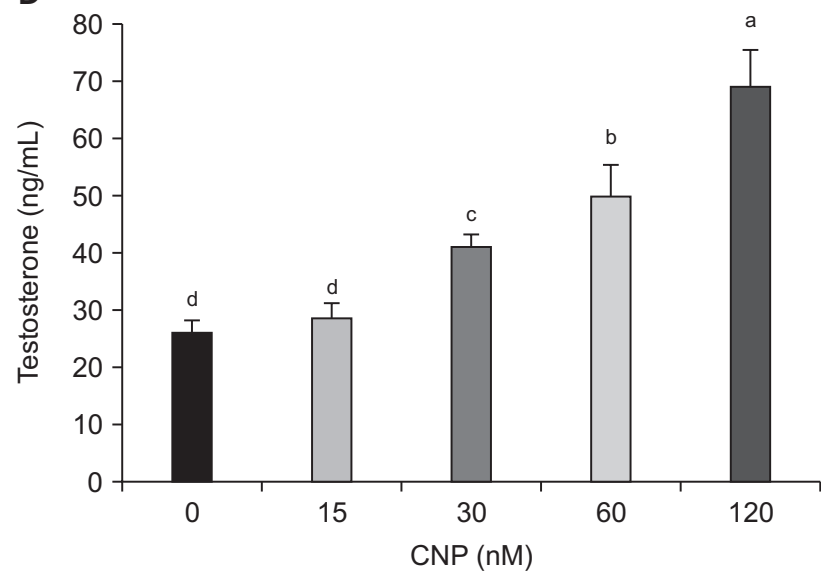

Fig. 2. Effects of C-type natriuretic peptide (CNP) on cyclic guanosine monophosphate (cGMP) production, natriuretic peptide receptor 2 (NPR2) expression, cell proliferation, testosterone secretion in mouse Leydig cells. (A) The cGMP production in mouse Leydig cells. (B) The NPR2 expression in mouse Leydig cells. (C) The cell activity in mouse Leydig cells. (D) The testosterone secretion in mouse Leydig cells. The $\beta$-actin was used as internal control. The data are presented as the mean \pm standard error of mean of three independent experiments. Bars with different letters are significantly different $(\mathrm{p}<0.05)$. 


\section{Natriuretic peptide receptor 2 knockdown} inhibits cyclic guanosine monophosphate production and cell proliferation in mouse

\section{Leydig cells}

To investigate role NPR2 on cell proliferation, the mouse Leydig cells were transfected with shRNANPR2 and shRNA-negative lentivirus. And the NPR2 knockdown or shRNA-negative mouse Leydig cells were treated with $30 \mathrm{nM}$ CNP for 24 hours. RT-qPCR and western blot showed that shRNA-NPR2 lentiviral effectively suppresses NPR2 expression in both mRNA and protein levels (Fig. 3A, 3B). Moreover, ELISA showed that NPR2 depleted Leydig cells have a lower expression levels of cGMP (Fig. 3C). CCK8 results indicated that a decreased cell viability is observed in NPR2 knockdown group compared with the control group (Fig. 3D). Flow cytometry analysis showed that the S phase of cells in the NPR2 knockdown group is higher compared the control group (Table 1). In addition, the mRNA expression of cell cycle factors (cyclin $\mathrm{A} 1$, cyclin B1, and cyclin D2) is downregulation in NPR2 knockdown mouse Leydig cells compared the control group (Fig. 3E).

Table 1. Cell cycle value comparison between shRNA-negative and shRNA-natriuretic peptide receptor 2

\begin{tabular}{lclc}
\multicolumn{1}{c}{ Group } & G1-phase (\%) & S-phase (\%) & G2-phase (\%) \\
\hline shRNA-negative & $63.37 \pm 2.35$ & $15.43 \pm 1.83$ & $21.23 \pm 2.49$ \\
shRNA-NPR2 & $57.33 \pm 1.49^{*}$ & $23.18 \pm 2.28^{*}$ & $19.67 \pm 1.34$ \\
\hline
\end{tabular}

Values are presented as mean \pm standard error of mean of three independent experiments. *An asterisk indicates the level of significance within the columns $(p<0.05)$.
A

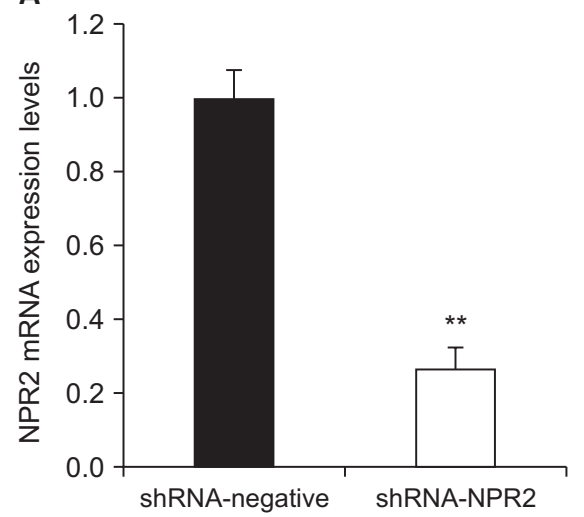

D

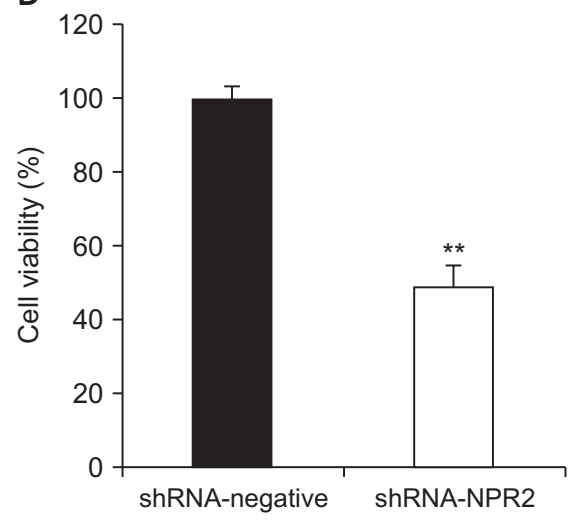

B

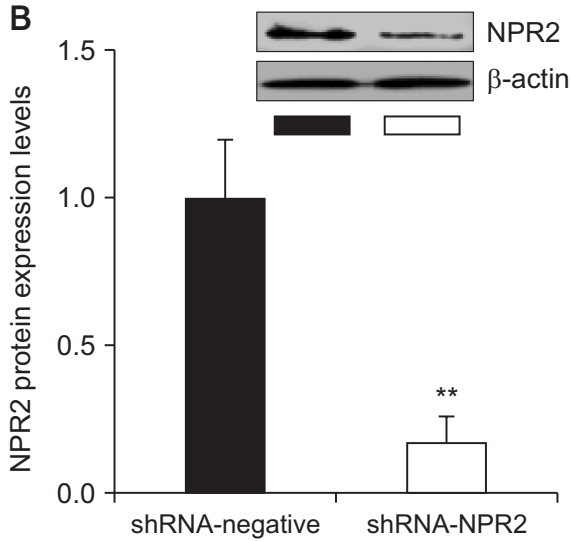

E

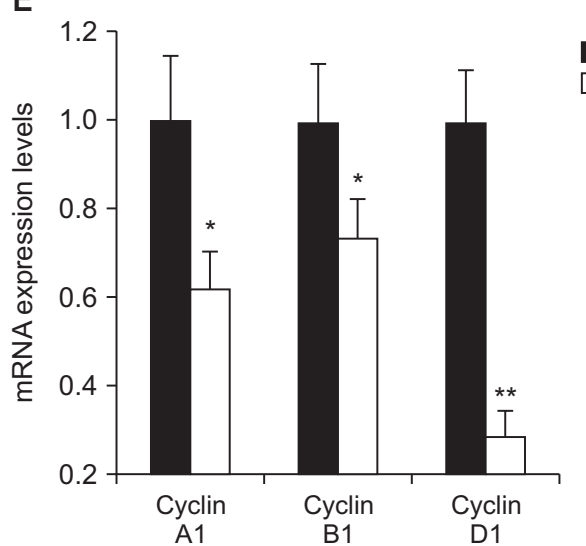

C

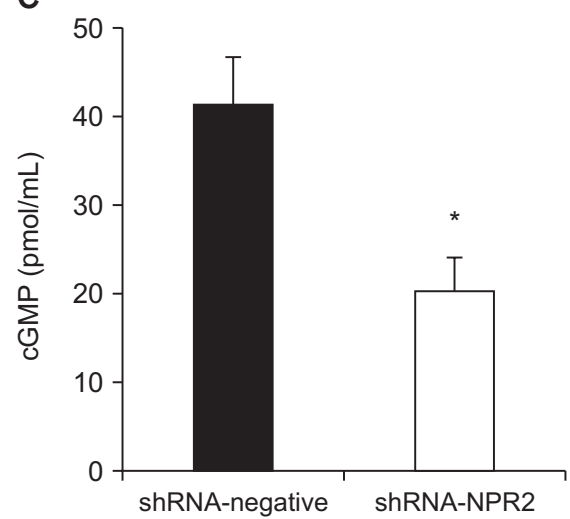

shRNA-negative ShRNA-NPR2

Fig. 3. Effects of natriuretic peptide receptor 2 (NPR2) knockdown on cyclic guanosine monophosphate (cGMP) production and cell proliferation in mouse Leydig cells. (A and B) The NPR2 expression in mRNA and Western blot levels in mouse Leydig transducted with shRNA-NPR2 lentivirus. (C) The cGMP production in mouse Leydig cells transducted with shRNA-NPR2 lentivirus. (D) The cell activity in mouse Leydig cells transducted with shRNA-NPR2 lentivirus. (E) The mRNA expression of cyclin A1, cyclin B1, and cyclin D2 in mouse Leydig cells transducted with shRNA-NPR2 lentivirus. The $\beta$-actin was used as internal control. The protein of NPR2 were normalized to that of $\beta$-actin. The results are presented as the mean \pm standard error of mean and are representative of three independent experiments. ${ }^{*} p<0.05$ and ${ }^{* *} p<0.01$ compared with each corresponding group in the control. 


\section{Natriuretic peptide receptor 2 knockdown} increases apoptosis in mouse Leydig cells

To determine the effect of NPR2 knockdown on mouse Leydig cells apoptosis, the cells were cultured in culture medium with $30 \mathrm{nM}$ CNP for 24 hours and detected by flow cytometry, RT-qPCR and Western blot. The results showed that the apoptosis rate of the shRNA-NPR2 group is significantly higher than shR-

Table 2. Cell apoptosis value comparison between shRNA-negative and shRNA-natriuretic peptide receptor 2

\begin{tabular}{llc}
\multicolumn{1}{c}{ Groups } & Live cells (\%) & Apoptosis cells (\%) \\
\hline shRNA-negative & $86.73 \pm 2.56$ & $13.03 \pm 1.08$ \\
shRNA-NPR2 & $72.38 \pm 1.18^{*}$ & $27.49 \pm 0.93^{*}$ \\
\hline
\end{tabular}

Values are presented as mean \pm standard error of mean of three independent experiments. ${ }^{*}$ An asterisk indicates the level of significance within the columns $(p<0.05)$.
NA-negative group (Table 2). Furthermore, the mRNA levels of apoptosis-related genes (p53, caspase-3, Bcl-2, Bax) were quantitatively analyzed. The mRNA levels of p53 and caspase-3 and Bax are obviously upregulated. However, the Bcl-2 expression is not altered significantly (Fig. 4A). Moreover, NPR2 knockdown increases the caspase-3 activity and the BAX protein expression, but not Bcl-2 (Fig. 4C, 4D). In addition, we also detected the phosphorylation of AKT, which is important for cell proliferation. The results showed that the pAKT of shRNA-NPR2 group is significantly decreased compared with shRNA-negative group (Fig. 4B).

\section{Natriuretic peptide receptor 2 knockdown decreased testosterone secretion in mouse Leydig cells}

To assess the role of NPR2 on steroid hormone lev-

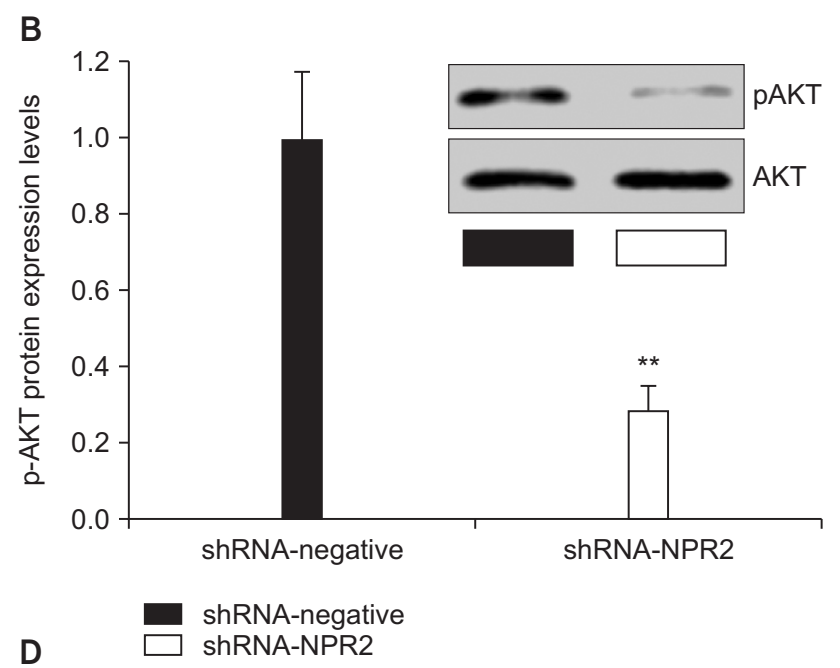

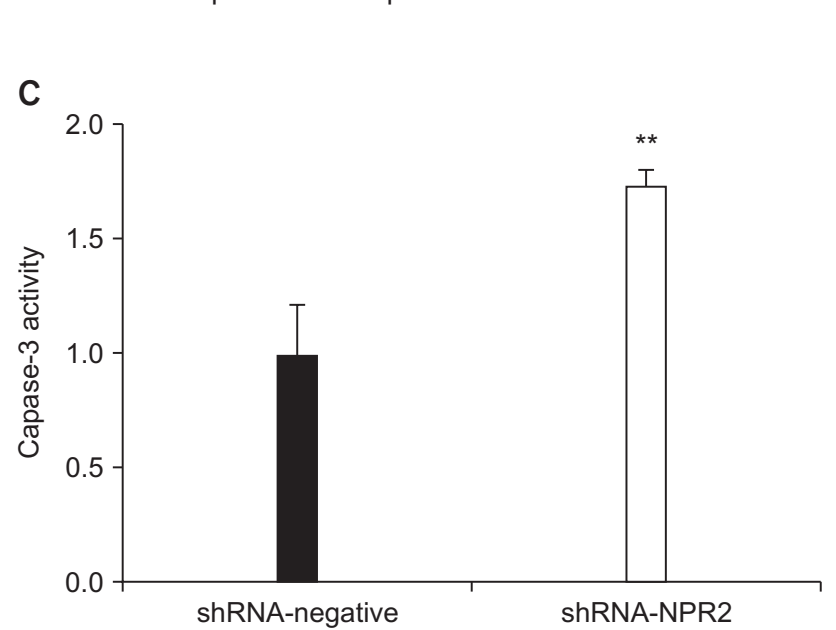

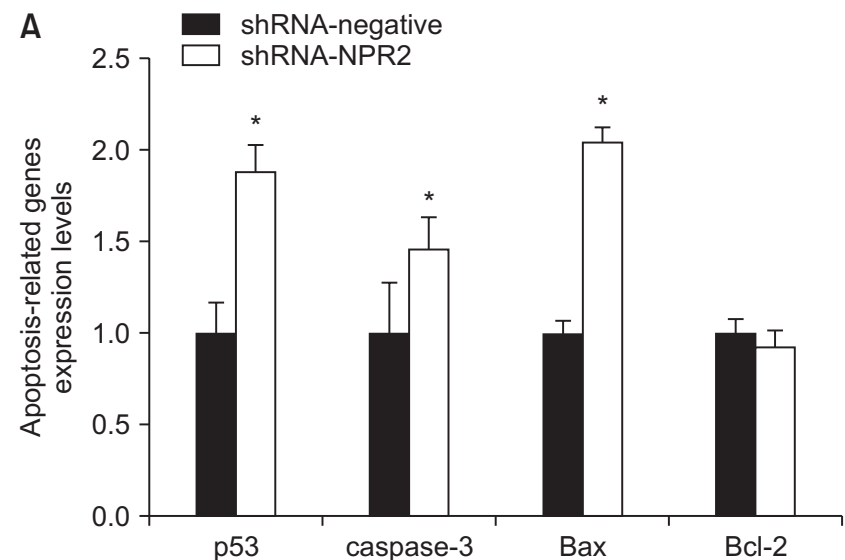

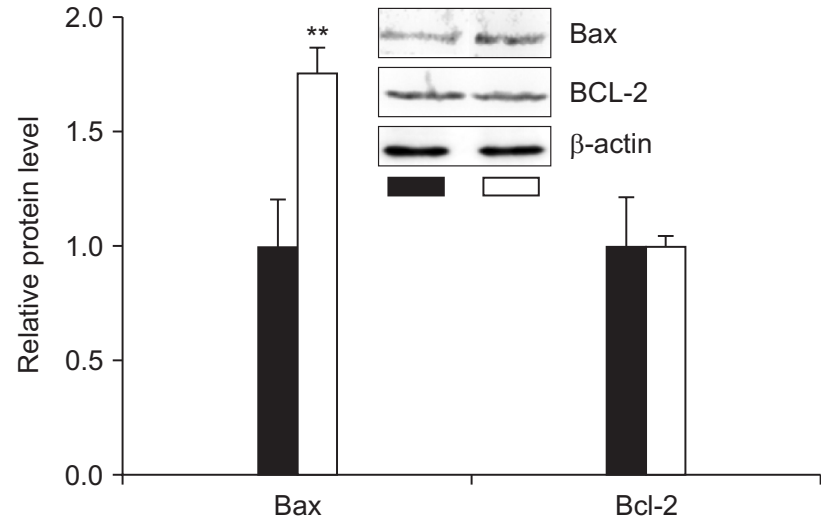

Fig. 4. Effects of natriuretic peptide receptor 2 (NPR2) knockdown on cells apoptosis in mouse Leydig cells. (A) Relative mRNA expression of cell apoptosis related genes (Bcl-2, Bax, p53, and Caspase-3) in mouse Leydig cells transducted with shRNA-NPR2 lentivirus. (B) The phosphorylation of AKT in mouse Leydig cells transducted with shRNA-NPR2 lentivirus. (C) Caspase-3 activity in mouse Leydig cells transducted with shRNA-NPR2 lentivirus. (D) Relative protein expression of Bcl-2 and Bax in mouse Leydig cells transducted with shRNA-NPR2 lentivirus. The $\beta$-actin was used as internal control. The phosphorylation of AKT were normalized to that of total AKT. The results are presented as the mean \pm standard error of mean and are representative of three independent experiments. ${ }^{*} \mathrm{p}<0.05$ and ${ }^{* *} \mathrm{p}<0.01$ compared with each corresponding group in the control. 
Men's Health

A

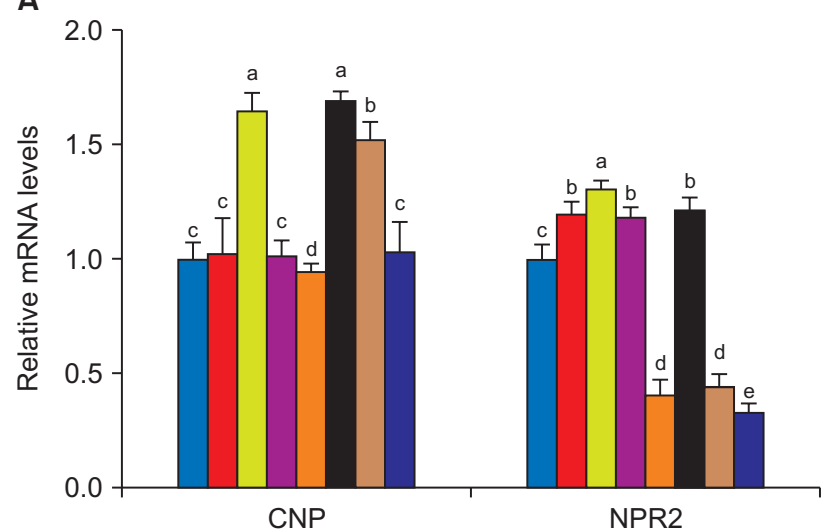

C

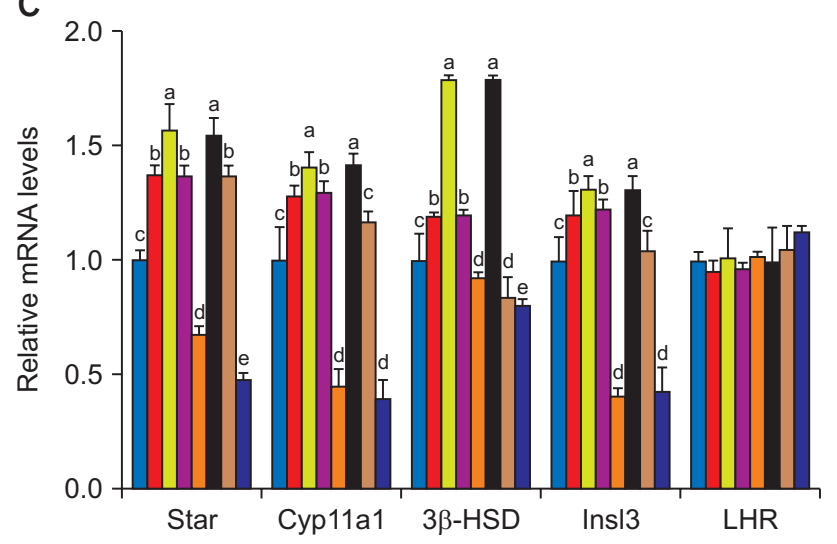

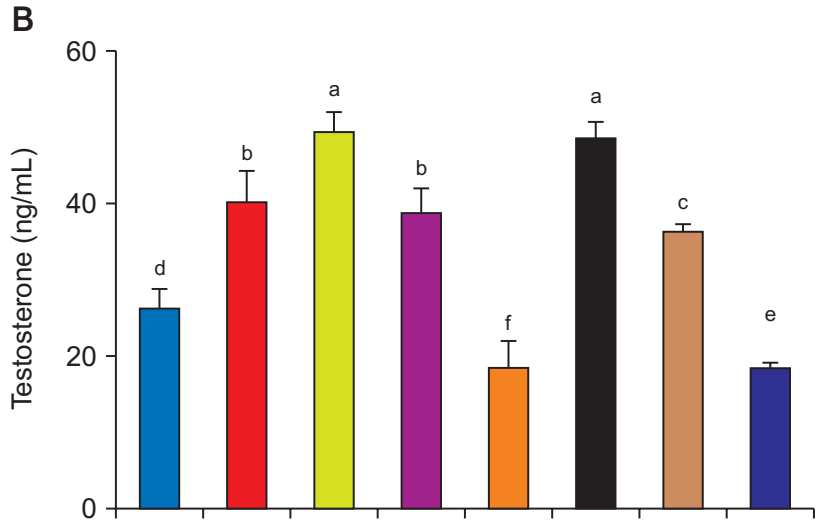

D

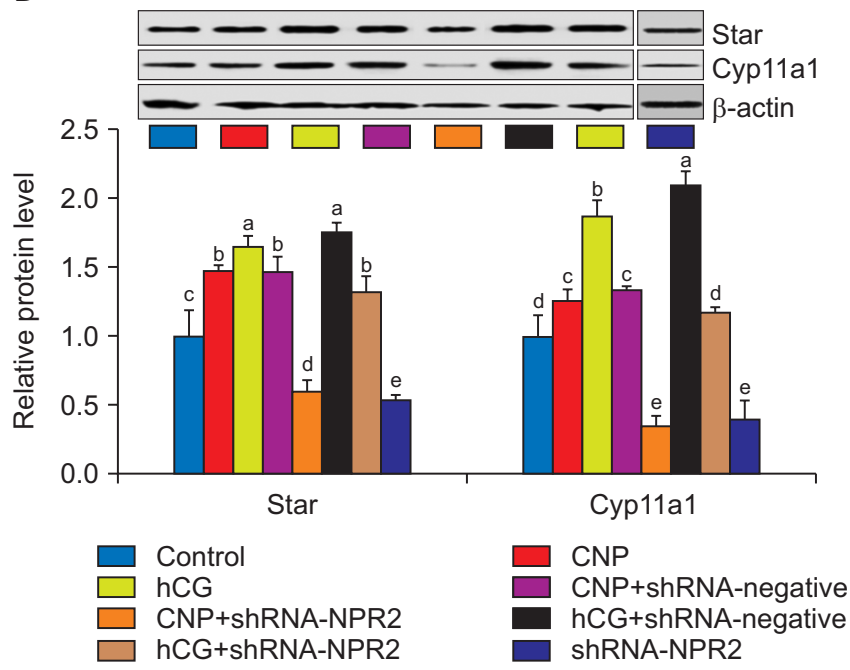

Fig. 5. Effect of natriuretic peptide receptor 2 (NPR2) knockdown on testosterone secretion in mouse Leydig cells. (A) The effects C-type natriuretic peptide (CNP) and human chorionic gonadotropin (hCG) on CNP/NPR2 expression. (B) Concentration of testosterone secretion in the culture medium transducted with shRNA-NPR2 lentivirus. (C) Relative mRNA expression of genes (Star, Cyp11a1, 3 $\beta$-hydroxysteroid dehydrogenase [33-HSD], insulin-like factor 3 [Insl3], and luteinizing hormone receptor [LHR]) in mouse Leydig cells transducted with shRNA-NPR2 lentivirus. (D) Relative protein expression of Star and Cyp11a1 in mouse Leydig cells transducted with shRNA-NPR2 lentivirus. The $\beta$-actin was used as internal control. The results are presented as the mean \pm standard error of mean and are representative of three independent experiments. Bars with different letters are significantly different $(p<0.05)$.

els, we measured the concentrations of testosterone in culture medium with $30 \mathrm{nM} \mathrm{CNP}$ or $2 \mathrm{IU}$ human chorionic gonadotropin (hCG) after cultured for 24 hours. The results showed that both CNP and hCG can promote testosterone secretion and the expression of steroidogenic enzymes (Fig. 5B-5D). Moreover, hCG seems to enhance the CNP/NPR2 expression (Fig. 5A). After transfection, the levels of testosterone are significantly lower in the shRNA-NPR2 group than negative group both in CNP and hCG treatment (Fig. 5B). We further analyzed the mRNA expression of several genes encoding steroidogenic enzymes, including Star, Cyp1la1, 3B-HSD, Insl3, and LHR. The result showed that the mRNA levels of Star, Cyp11a1, Insl3, and 33-HSD sig- nificantly decreased, and the protein levels of Star and Cyp1la1 also induced by CNP and hCG, whereas LHR are no obvious change in mRNA level (Fig. 5C, 5D).

\section{The mechanism of C-type natriuretic peptide, testosterone and estradiol in natriuretic peptide receptor 2 expression in mouse Leydig cells}

Because testosterone promotes NPR2 expression directly via androgen receptor (AR) or indirectly via estrogen receptor (ER) by promoting estrogen production [15], and Leydig cells can convert a bit of testosterone to estrogen. We hypothesized that CNP promotes NPR2 expression via $\mathrm{AR}$ or $\mathrm{ER}$ in mouse Leydig cells. To test 
A

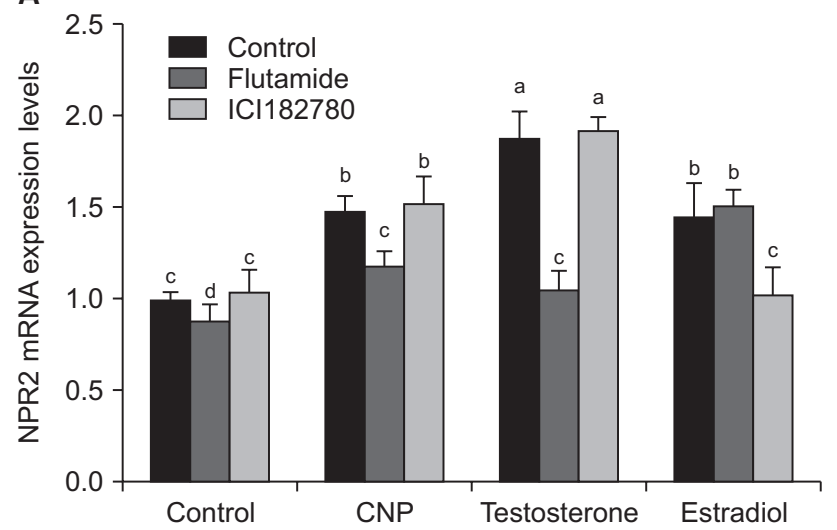

B

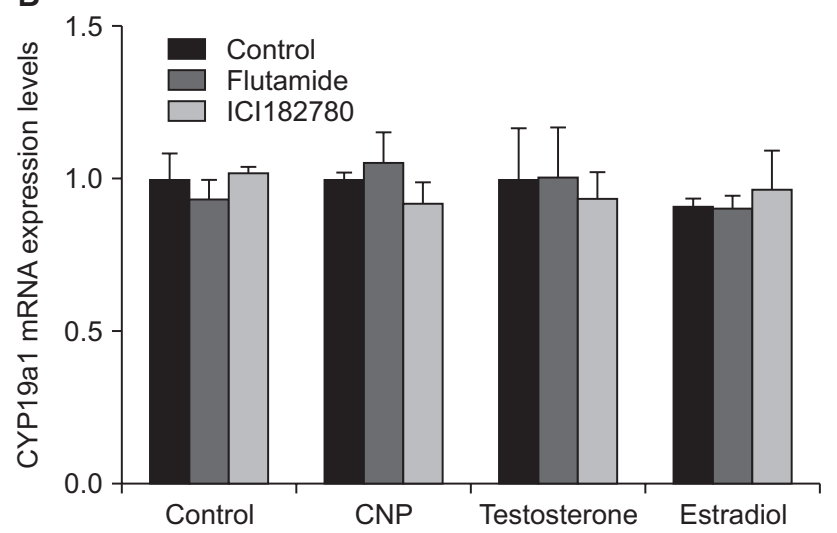

Fig. 6. The mechanism of C-type natriuretic peptide (CNP), testosterone, and estradiol in promoting natriuretic peptide receptor 2 (NPR2) expression in mouse Leydig cells. (A) The expression of NPR2 after stimulation with CNP, testosterone and estradiol with or without flutamide and ICl182780 in mouse Leydig cells. (B) The expression of Cyp19a1 after stimulation with CNP, testosterone and estradiol with or without flutamide and ICl182780 in mouse Leydig cells. The $\beta$-actin was used as internal control. The data are presented as the mean \pm standard error of mean of three independent experiments. Bars with different letters are significantly different $(p<0.05)$.

this hypothesis, the mouse Leydig cells were treated with $30 \mathrm{nM}$ CNP, $30 \mathrm{nM}$ testosterone, or $30 \mathrm{nM}$ estrogen in the presence or absence of $500 \mathrm{nM}$ AR inhibitor flutamide and $500 \mathrm{nM}$ ER inhibitor ICI182780 for 24 hours during the culture.

Results showed that flutamide partly inhibits the CNP- and testosterone-induced NPR2 expression in mouse Leydig cells, but no obvious effects on estrogeninduced NPR2 expression (Fig. 6A). At the same time, ICI182780 successfully inhibits the expression of NPR2 induced by estrogen, but no obvious changes is observed in other groups (Fig. 6A). We also detected the CYP19a1 (the enzyme responsible for androgen aromatization to estrogen) expression by RT-qPCR. The results showed that all the treatment have no obvious effects on CYP19a1 expression (Fig. 6B).

\section{DISCUSSION}

Numerous studies have demonstrated that CNP/ NPR2 plays an important role in female reproduction. However, the functions of CNP/NPR2 in male reproduction, especially in Leydig cells, remain poorly understood. In this research, we aimed to study the role of CNP/NPR2 on cGMP production, cell cycle, apoptosis, testosterone secretion and related regulatory mechanism in mouse Leydig cells.

We first detected NPR2 location in male mouse testis and found NPR2 is mainly expressed in Leydig cells. As far as know, this is the first report that NPR2 is mainly expressed in mouse Leydig cells. We also found that CNP was expressed in mouse Leydig cells, this is similar with the previous studies in rat [13,14]. We also detected CNP/NPR2 expression from born to sexual maturity and showed age-related increase, although the expression level of CNP/NPR2 is higher at birth. Higher expression levels of CNP/NPR2 at birth maybe that:1) CNP/NPR2 may play an important role in fetal development, especially the rapid growth of the fetus (CNP/NPR2 is a key regulatory factor for bone growth) [16,17]; 2) Higher expression levels of CNP/NPR2 on 0 day indicated that they may have an intimate relation ship with the onset of spermatogenesion [8]. This results are further supported by a previous study in rat [7].

The change in CNP/NPR2 expression may imply that CNP/NPR2 may be involved in the testicular development, testosterone secretion, spermatogenesis in the male reproduction as previous reports $[7,8,13,18]$. As NPR2 is expressed in Leydig cells of male mice testes and the binding CNP and NPR2 generates cGMP to regulate cell function. So, we used Leydig cells as cell model and added CNP in the culture medium to active CNP/NPR2 signaling pathway during the following research. We found that CNP promotes cGMP production in Leydig cells, suggesting that NPR2 is successfully active by CNP.

The effect of CNP/NPR2 signaling pathway on Leydig cell proliferation and cycle have never reported. Our study revealed that CNP enhances cell proliferation in a dose-dependent manner. NPR2 knockdown significantly reduces the proliferation activity and the 
cells are arrested at the $\mathrm{S}$ phase. This may be because $\mathrm{CNP} / \mathrm{NPR} 2$ is related with expression of cell cycle factors (cyclin A1, cyclin B1, and cyclin D2). Cyclin A1 and cyclin D2 are key regulators associated with the S-toG2/M transition. Moreover, cyclin B1 is an important gene related to regulating the normal cell-cycle progression and plays a vital role in mitotic exit and the beginning of the subsequent cell division. Thus, the cell-cycle results imply that CNP/NPR2 is involved in the growth and proliferation in Leydig cells. In addition, some studies report that CNP is a growth inhibitor in smooth muscle cells [19,20], and some studies reported that CNP promotes the growth of the bone [17]. This difference may be related to cell types or the indirect effects of CNP, such as testosterone synthesis [21] and insulin-like growth factor I production [16].

Previous study showed that NPR2 gene knockout mice have smaller size [16] and smaller testis [8] compared with the normal mice. However, whether CNP/ NPR2 regulates mouse Leydig cells viability and apoptosis remains unclear. In the current study, we found that CNP effectively promotes the viability of mouse Leydig cells. At the same time, NPR2 depletion remarkably induces apoptosis in mouse Leydig cells. To clarify this result further, we measured the expression of key molecules associated with apoptosis via RTqPCR and Western blot. As an important transcription factor, p53 can promote cell apoptosis, and elevated p53 mRNA levels result in higher apoptosis rates in Leydig cells [22]. Caspase-3, acts as an executioner in caspasemediated apoptosis, is extensively involved various cell apoptosis. Moreover, in the Bcl-2 family, Bcl-2 plays an anti-apoptotic role, whereas Bax has a proapoptotic effect [23,24]. In the present study, the expression of Bax, p53 and caspase-3 was significantly upregulated after NPR2 disruption, whereas the expression Bax levels appeared unchanged. Based on these results, we concluded that the apoptosis cells in mouse Leydig cells caused by disrupting NPR2 is closely correlated with these molecules. In addition, PI3K-AKT pathway plays an key role in cell proliferation and survival [25] and NPR2 knockdown inhibits the activation of AKT in in mouse Leydig cells. This also showed that NPR2 regulates cells viability and apoptosis in another perspective.

Given the importance of Leydig cells in secretion of testosterone, we evaluated the CNP/NPR2 signaling pathway on testosterone synthesis in mouse Leydig cells. Our results indicated that CNP promotes testosterone secretion in mouse Leydig cells as a previous report [21] and NPR2 silencing inhibits testosterone production. The possible reason for the reductions of testosterone could be the decreases in the Star, Cyp11a1 and 33-HSD, which is essential for testosterone biosynthesis $[4,26]$. Moreover, the downregulation of cGMP may also inhibits testosterone production [27]. However, the expression levels of LHR was no obvious after NPR2 knockdown in Leydig cells. In addition, we also found that activated LH signal by hCG induces the CNP/NPR2 expression and NPR2 disruption partly reduces the hCG-induced testosterone secretion. This result suggested that CNP/NPR2 signal maybe related with LHR signal during the testosterone secretion. Thus, we inferred that CNP/NPR2 may play an important role in the development of male reproductive tissues as well as promoting secondary sexual characteristics, such as increased muscle and bone mass by promoting testosterone production.

We also found that CNP promotes NPR2 expression in mouse Leydig cells. And previous studies report that testosterone enhances NPR2 expression in cumulus cell $[15,28]$. So, we hypothesized that CNP-induced NPR2 expression is by promoting testosterone production, which further enhances NPR2 expression via AR in mouse Leydig cells. Consequently, we inhibited AR by flutamide during the culture with CNP and testosterone. The result that inhibited AR by flutamide partially improves NPR2 expression in Leydig cells. We therefore speculated that testosterone is an important factor in regulating CNP/NPR2 in Leydig cells as previous report in ovary [15]. As Leydig cells can convert a bit of testosterone to estrogen [29] and estrogen promotes NPR2 expression via ER [28,30], we detected the role of ER on CNP- or testosterone-induced NPR2 expression. We found inhibited ER by ICI182780 has no obvious effects on NPR2 expression in mouse Leydig cells and this result is further supported by the result of CYP19a1 mRNA expression. These results indicated that CNP promotes testosterone secretion, which further induces NPR2 expression via AR in mouse Leydig cells.

\section{CONCLUSIONS}

Our study highlights the important role of CNP/ NPR2 in mouse testes. We found that CNP/NPR2 is re- 
lated with cell proliferation and testosterone secretion in mouse Leydig cells. In addition, CNP can promote NPR2 expression via AR in mouse Leydig cells. Our findings not only provided new insights into the function of CNP/NPR2 in female reproduction but also provide potential therapeutic targets for treating reduced testosterone.

\section{ACKNOWLEDGEMENTS}

This research study was funded by the National Natural Science Foundation of China (No. 81660535), Project of Science and Technology of Jiangxi Provincial Education Department (GJJ170966), and Doctoral Research Start-Up Foundation of Jiujiang University (No. 8879522).

\section{Disclosure}

The authors have no potential conflicts of interest to disclose.

\section{Author Contribution}

Conceptualization: Yang L. Data curation: Huang S. Formal analysis: Yang L. Funding acquisition: Yang L, Huang S. Investigation: Lei L, Zhao Q. Methodology: Yang L, Gong Y, Guan G. Project administration: Huang S. Resources: Huang S. Software: Lei L, Guan G. Supervision: Gong Y. Validation: Yang L. Visualization: Yang L, Zhao Q. Writing-original draft: Lei L. Writingreview \& editing: Yang L.

\section{REFERENCES}

1. Ito A, Shirakawa H, Takumi N, Minegishi Y, Ohashi A, Howlader $\mathrm{ZH}$, et al. Menaquinone-4 enhances testosterone production in rats and testis-derived tumor cells. Lipids Health Dis $2011 ; 10: 158$.

2. Huhtaniemi IT. LH and FSH receptor mutations and their effects on puberty. Horm Res 2002;57 Suppl 2:35-8.

3. Ferlin A, Bogatcheva NV, Gianesello L, Pepe A, Vinanzi C, Agoulnik AI, et al. Insulin-like factor 3 gene mutations in testicular dysgenesis syndrome: clinical and functional characterization. Mol Hum Reprod 2006;12:401-6.

4. Tremblay JJ. Molecular regulation of steroidogenesis in endocrine Leydig cells. Steroids 2015;103:3-10.

5. Zhang M, Su YQ, Sugiura K, Xia G, Eppig JJ. Granulosa cell ligand NPPC and its receptor NPR2 maintain meiotic arrest in mouse oocytes. Science 2010;330:366-9.
6. Yang L, Wei Q, Li W, Ge J, Zhao X, Ma B. C-type natriuretic peptide improved vitrified-warmed mouse cumulus oocyte complexes developmental competence. Cryobiology 2016;72: 161-4.

7. Huang DH, Zhang SW, Zhao H, Zhang L. The role of C-type natriuretic peptide in rat testes during spermatogenesis. Asian J Androl 2011;13:275-80.

8. Sogawa C, Fujiwara Y, Tsukamoto S, Ishida Y, Yoshii Y, Furukawa $\mathrm{T}$, et al. Mutant phenotype analysis suggests potential roles for C-type natriuretic peptide receptor (NPR-B) in male mouse fertility. Reprod Biol Endocrin 2014;12:64.

9. Kong N, Xu X, Zhang Y, Wang Y, Hao X, Zhao Y, et al. Natriuretic peptide type $C$ induces sperm attraction for fertilization in mouse. Sci Rep 2017;7:39711.

10. Xia H, Chen Y, Wu KJ, Zhao H, Xiong CL, Huang DH. Role of C-type natriuretic peptide in the function of normal human sperm. Asian J Androl 2016;18:80-4.

11. Yang L, Wei Q, Li W, Xi Q, Zhao X, Ma B. NPR2 is involved in FSH-mediated mouse oocyte meiotic resumption. J Ovarian Res 2016;9:6.

12. Chen H, Gao L, Xiong Y, Yang D, Li C, Wang A, et al. Circadian clock and steroidogenic-related gene expression profiles in mouse Leydig cells following dexamethasone stimulation. Biochem Bioph Res Co 2017;483:294-300.

13. Xia W, Mruk DD, Cheng CY. C-type natriuretic peptide regulates blood-testis barrier dynamics in adult rat testes. Proc Natl Acad Sci USA 2007;104:3841-6.

14. Collin O, Lissbrant E, Bergh A. Atrial natriuretic peptide, brain natriuretic peptide and c-type natriuretic peptide: effects on testicular microcirculation and immunohistochemical localization. Int J Androl 1997;20:55-60.

15. Wang X, Wang H, Liu W, Zhang Z, Zhang Y, Zhang W, et al. High level of C-type natriuretic peptide induced by hyperandrogen-mediated anovulation in polycystic ovary syndrome mice. Clin Sci (Lond) 2018;132:759-76.

16. Tsuji T, Kunieda T. A loss-of-function mutation in natriuretic peptide receptor 2 (Npr2) gene is responsible for disproportionate dwarfism in $\mathrm{cn} / \mathrm{cn}$ mouse. J Biol Chem 2005;280: 14288-92.

17. Mericq V, Uyeda JA, Barnes KM, De LF, Baron J. Regulation of fetal rat bone growth by C-type natriuretic peptide and cGMP. Pediatr Res 2000;47:189-93.

18. Karan S, Frederick JW, Baehr W. Novel functions of photoreceptor guanylate cyclases revealed by targeted deletion. Mol Cell Biochem 2010;334:141-55.

19. Porter JG, Catalano R, Mcenroe G, Lewicki JA, Protter AA. C-type natriuretic peptide inhibits growth factor-dependent DNA synthesis in smooth muscle cells. Am J Physiol 1992; 


\section{Men's Health}

263:C1001-6.

20. Furuya M, Yoshida M, Hayashi Y, Ohnuma N, Minamino N, Kangawa $\mathrm{K}$, et al. C-type natriuretic peptide is a growth inhibitor of rat vascular smooth muscle cells. Biochem Biophys Res Commun 1991;177:927-31.

21. Khurana ML, Pandey KN. Receptor-mediated stimulatory effect of atrial natriuretic factor, brain natriuretic peptide, and C-type natriuretic peptide on testosterone production in purified mouse Leydig cells: activation of cholesterol side-chain cleavage enzyme. Endocrinology 1993;133:2141-9.

22. Fouchécourt S, Livera G, Messiaen S, Fumel B, Parent AS, Marine JC, et al. Apoptosis of sertoli cells after conditional ablation of murine double minute $2(\mathrm{Mdm} 2)$ gene is p53-dependent and results in male sterility. Cell Death Differ 2016; 23:521-30.

23. Zhao F, Wang N, Yi Y, Lin P, Tang K, Wang A, et al. Knockdown of CREB3/Luman by shRNA in mouse granulosa cells results in decreased estradiol and progesterone synthesis and promotes cell proliferation. Plos One 2016;11:e0168246.

24. Sifer C, Bénifla JL, Bringuier AF, Porcher R, Blanc-Layrac G, Madélénat $\mathrm{P}$, et al. Could induced apoptosis of human granulosa cells predict in vitro fertilization-embryo transfer outcome? A preliminary study of 25 women. Eur J Obstet Gyn R
Lei Yang, et al: CNP/NPR2 Promotes Testosterone Production

B 2002;103:150-3.

25. Datta SR, Dudek H, Tao X, Masters S, Fu H, Gotoh Y, et al. Akt phosphorylation of bad couples survival signals to the cell-intrinsic death machinery. Cell 1997;91:231-41.

26. Hatano M, Migita T, Ohishi T, Shima Y, Ogawa Y, Morohashi KI, et al. SF-1 deficiency causes lipid accumulation in Leydig cells via suppression of STAR and CYP11A1. Endocrine 2016; 54:484-96.

27. Moon HW, Park JW, Lee KW, Jeong HC, Choi JB, Choi SW, et al. Administration of Goji (lycium Chinense mill.) Extracts improves erectile function in old aged rat model. World J Mens Health 2016;35:43-50.

28. Zhang M, Su YQ, Sugiura K, Wigglesworth K, Xia G, Eppig JJ. Estradiol promotes and maintains cumulus cell expression of natriuretic peptide receptor 2 (npr2) and meiotic arrest in mouse oocytes in vitro. Endocrinology 2011;152:4377-85.

29. Abney TO. The potential roles of estrogens in regulating Leydig cell development and function: a review. Steroids 1999; 64:610-7.

30. Zhang J, Wei Q, Cai J, Zhao X, Ma B. Effect of C-type natriuretic peptide on maturation and developmental competence of goat oocytes matured in vitro. Plos One 2015;10:e0132318. 
Appendix. Primer sequences used for RT-qPCR

\begin{tabular}{|c|c|c|}
\hline Gene & Forward primer sequences $\left(5^{\prime} \rightarrow 3^{\prime}\right)$ & Reverse primer sequences $\left(5^{\prime} \rightarrow 3^{\prime}\right)$ \\
\hline CNP & GGTCTGGGATGTTAGTACAGCTA & TAAAAGCCACATTGCGTTGGA \\
\hline NPR2 & GCTGACCCGGCAAGTTCTGT & ACAATACTCGGTGACAATGCAGAT \\
\hline Cyclin A1 & GCCTTCACCATTCATGTGGAT & TTGCTGCGGGTAAAGAGACAG \\
\hline Cyclin B1 & AAGGTGCCTGTGTGTGAACC & GTCAGCCCCATCATCTGCG \\
\hline Cyclin D2 & TTACCTGGACCGCTTCTTG & GAGGCTTGATGGAGTTGTCG \\
\hline $\mathrm{Bcl} 2$ & GGTCATGTGTGTGGAGAGCGTCA & CTTCAGAGACAGCCAGGAGAAAT \\
\hline Bax & AGGATGCGTCCACCAAGAA & CAAAGTAGAAGAGGGCAACCAC \\
\hline Caspase-3 & TGACTGGAAAGCCGAAACTC & GCAAGCCATCTCCTCATCAG \\
\hline p53 & TACAAGAAGTCACAGCACAT & GATAGGTCGGCGGTTCAT \\
\hline Star & CCCACCGCATGGTCGAGTAGTG & CGCCTCTGCAGCCAACTCATG \\
\hline Cyp11a1 & ACACTGAGACTCCACCCCAT & CTCGACCCATGGCAAAGCTA \\
\hline Insl3 & TGCAGTGGCTAGAGCAGAGA & GTGCAGCCAGTAAGACAGCA \\
\hline Cyp19a1 & GCACAGTCACTACATCTCCCGA & CACACAAACTTCCACCATTCGA \\
\hline LHR & AGAGTGATTCCCTGGAAAGGA & CTTACGAAAGGTTCCCTACT \\
\hline $3 \beta-H S D$ & TGGACAAAGTATTCCGACCAGA & GGCACACTTGCTTGAACACAG \\
\hline$\beta$-actin & GCAAGCAGGAGTACGATGAG & CCATGCCAATGTTGTCTCTT \\
\hline
\end{tabular}

The $\beta$-actin was used as internal control. 